\title{
Die Schönheit reden. Überlegungen nach Anton Raphael Mengs und Johann Joachim Winckelmann
}

Problem, jak ująć w słowa sferę sztuki, był w refleksjach estetycznych wieku osiemnastego jednym z centralnych i bardzo żywo dyskutowanych tematów. Indywidualny charakter oglądu dzieł sztuki, bazujący na doznaniach uczuciowych i jednocześnie przenikliwy intelektualnie, budzi poczucie zmysłowej bliskości w kontakcie ze sztuką, niedoświadczane do tej pory w podobnym stopniu. Doznania te stają się wyzwaniem nie tylko dla nowatorskich prób opisu obrazu, lecz również wpływają na literaturę teoretyczną, która w wieloraki sposób przekracza dotychczasowe dyskursywne kategorie.

Poniższy szkic omawia elementy zmian języka literatury teoretycznej poświęconej sztuce na przykładzie wczesnych pism Johanna Joachima Winckelmanna i Antona Raphaela Mengsa. Na szczególną uwagę w tekstach tych zasługują związki między retoryką i oglądem dzieł sztuki.

Die Mitteilbarkeit von Kunsterfahrung bildet ein zentrales und lebhaft diskutiertes Problem in den ästhetischen Reflexionen des 18. Jahrhunderts. Die individuelle, gefühlsbetonte und zugleich scharfsinnige Kunstbetrachtung erzeugt eine bisher kaum in solchem Grade wahrgenommene sinnliche Nähe zum Kunstobjekt. Diese Erfahrung wird zur Herausforderung nicht nur für die Bildbeschreibung, sondern auch für die theoretische Kunstliteratur, die in vielerlei Hinsicht aus ihrer diskursiven Enge ausbricht.

Der Beitrag beschäftigt sich mit den sprachlichen Veränderungen in den theoretischen Erörterungen am Beispiel von frühen Schriften von Johann Joachim Winckelmann und Anton Raphael Mengs. Die enge Verbindung zwischen der Redekunst und der Kunstbeschreibung kommt dabei besonders zum Vorschein.

The 18th century saw a shift in the meaning of art. The communicability of art experience was a central and lively discussed problem in the aesthetic reflections of the 18th century. The individual, emotional and yet astute viewing of art produced a sensual 
proximity to the artwork hardly noticed so far. This experience was a challenge not only for the description of pictures, but also for the theoretical literature on art.

This essay deals with the changes of style in theoretical literature on art on the example of the early writings of Johann Joachim Winckelmann and Anton Raphael Mengs. Particularly, the close connection between rhetoric and the description of art will be shown.

Nach der Ankunft Winckelmanns (1717-1768) in Rom im November 1755 wurden ihm die Betrachtung und Beschreibung der Kunstwerke zum ersten bedeutenden Anliegen. Vor Ort, als er die antiken Originale zu Gesicht bekommt, empfindet er besonders intensiv die Unzulänglichkeiten der etablierten Kunstliteratur: „Aus Beschreibungen der übrigen Alterthümer, der Gallerien und Villen zu Rom, ist eben so wenig Unterricht für die Kunst zu ziehen; sie verführen mehr als sie unterrichten“, resümiert er später in der Vorrede zur Geschichte der Kunst des Alterthums 1763 (WINCKELMANN 1968d:238). „Ueberhaupt sind die mehrsten Scribenten in diesen Sachen, wie die Flüsse, welche aufschwellen, wenn man ihr Wasser nicht nöthig hat, und trocken bleiben, wenn es am Wasser fehlt.“ (WINCKELMANN 1968d:243) Die gewöhnliche Flüchtigkeit der Anschauung, aber auch das Unvermögen des sprachlichen Ausdrucks geben Winckelmann einen wichtigen Impuls, selbst tätig zu werden, und ändern die Ausrichtung seines zuerst auf das „Sehen“ angelegten Aufenthalts zu einer epochemachenden Schaffensphase (vgl. SCHRÖTER 1986:55).

Der Dresdner Hofmaler Anton Raphael Mengs (1728-1779) ${ }^{1}$, der sich seit 1751 ebenfalls dauerhaft in Rom aufhielt, wurde Winckelmann zum wichti-

1 Anton Raphael Mengs wurde 1728 in Aussig in Böhmen geboren, als Sohn des Miniaturmalers Ismael Mengs, der seit 1714 in Dresden als Hofmaler tätig war. Anton Raphael Mengs wurde mit knapp siebzehn Jahren ebenfalls zum Hofmaler in Dresden ernannt, lebte und arbeitete jedoch seit 1751 vor allem in Rom, wo er ab 1754 an der Accademia Capitolina unterrichtete und seit 1761 am spanischen Hof. Wegen Krankheit kehrte er 1777 aus Madrid dauerhaft nach Rom zurück, wo er 1779 starb. Mengs gilt als Wegbereiter des Klassizismus, genoss hohes Ansehen als einer der größten lebenden Künstler seiner Zeit. Mit seinen sehr populären theoretischen Schriften verdiente er sich bei den Zeitgenossen den Titel des „Malerphilosophen“ (vgl. SuTTER 1968). Nach 1800 geriet Mengs in Vergessenheit, heutzutage wird seine Bedeutung wieder erkannt. - Der Beitrag entstand im Rahmen des Projekts „SPOLEČENSKÉ, KULTURNÍ A IDEOVÉ TRANSFERY V HISTORII PŘÍHRANIČNÍHO REGIONU SEVEROZÁPADNÍCH A SEVERNÍCH ČECH A JEJICH ROLE PRO JEHO ROZVOJ A UDRŽITELNOST“, unterstützt vom Kultusministerium der Tschechischen 
gen Begleiter und Mentor auf diesem Wege. Sie blieben in engem Kontakt bis 1761, als Mengs nach Madrid geht. ${ }^{2}$ Winckelmann ermutigte den hoch geschätzten Maler erfolgreich, seine Philosophie zu Papier zu bringen, feierte ihn in der Geschichte der Kunst des Alterthums als neuen „,deutschen Raphael in Rom“ “3 , und die Vorrede schließt er mit den Worten: „Diese Geschichte der Kunst weihe ich der Kunst, und der Zeit, und besonders meinem Freunde, Herrn Anton Raphael Mengs.“ (WINCKELMANN 1968d:246) Goethe war einer der ersten, die die Bedeutung dieses Zusammentreffens zwischen dem theoretisch interessierten Künstler und dem (zu Beginn seines römischen Aufenthaltes überwiegend) Buchgelehrten Winckelmann erkannten:

Aber W. hätte lange Zeit in den weiten Kreisen altertümlicher Überbleibsel nach den wertesten, seiner Betrachtung würdigsten Gegenständen umhergetastet, hätte das Glück ihn nicht sogleich mit Mengs zusammengebracht. Dieser, dessen eigenes großes Talent auf die alten und besonders die schönen Kunstwerke gerichtet war, machte seinen Freund sogleich mit dem Vorzüglichsten bekannt, was unserer Aufmerksamkeit wert ist. Hier lernte dieser die Schönheit der Formen und ihrer Behandlung kennen, und sah sich sogleich aufgeregt, eine Schrift vom Geschmack der griechischen Künstler zu unternehmen. (GOETHE 1998a:191)

Am Rande von Winckelmanns kunstgeschichtlichen Studien, jedoch als deren bedeutende Grundlage, wird das Anschauen und Empfinden des Schönen

Republik und realisiert an der Philosophischen Fakultät der Universität Ústí nad Labem.

2 Über die Anfänge der Freundschaft und Zusammenarbeit vgl. RÖTTGEN (1986) und TRAutweIN (1997:93): „Während seiner Dresdner und frühen römischen Zeit, eigentlich eine Übergangsphase seiner wissenschaftlichen Biographie, war er [Winckelmann] noch auf die Hilfe Fremder, auf die künstlerischen Eingebungen eines Malers wie Mengs, angewiesen. Eine Art symbiotischer Beziehung hat man sich hier wohl vorzustellen.“

3 „Der Inbegriff aller beschriebenen Schönheiten in den Figuren der Alten, findet sich in den unsterblichen Werken Herrn Anton Raphael Mengs, ersten Hofmalers der Könige von Spanien und von Pohlen, des größten Künstlers seiner, und vielleicht auch der folgenden Zeit. Er ist als ein Phoenix gleichsam aus der Asche des ersten Raphaels erweckt worden, um der Welt in der Kunst die Schönheit zu lehren, und den höchsten Flug menschlicher Kräfte in derselben zu erreichen. Nachdem die Deutsche Nation stolz seyn konnte über einen Mann, der zu unserer Väter Zeiten die Weisen erleuchtet, und Saamen von allgemeiner Wissenschaft unter allen Völkern ausgestreuet, so fehlete noch an dem Ruhme der Deutschen, einen Wiederhersteller der Kunst aus ihrem Mittel aufzuzeigen, und den deutschen Raphael in Rom selbst, dem Sitz der Künste, dafür erkannt und bewundert zu sehen.“ (WINCKELMANN 1764:184). 
Gabriela Brudzyńska-Němec

zum Gegenstand der Reflexion. Nach der berühmten ersten Schrift aus dem Jahre 1755 Gedancken über die Nachahmung der Griechischen Wercke in der Mahlerey und Bildhauer-Kunst entstehen während des römischen Aufenthaltes kleinere pädagogisch und praktisch motivierte, theoretische Texte, die teilweise bis heute wenig diskutiert wurden (KASE 2010:233): Erinnerung über die Betrachtung der Werke der Kunst (1759) und Abhandlung von der Fähigkeit der Empfindung des Schönen in der Kunst, und dem Unterrichte in derselben (1763).

Aus der sorgfältigen Anschauung der Originale, die Winckelmann immer wieder deutlich herausstellt, um sich einerseits von der ungenauen Betrachtung der Kunstwerke und ebenso vom bloßen Bücherstudium zu distanzieren, geht auch eine neue Sprache der Kunstliteratur hervor. Das geschieht nicht von ungefähr, als spontanes Ergebnis einer auf die sinnliche Einfühlung orientierten Betrachtung, das eine fachmännische analytische Beschreibung des Kunstwerks oder eben die beifälligen Künstlerviten ablöst. Es handelt sich um eine vielförmige, für die ästhetische Wahrnehmung und sprachliche Sensibilität produktive Annäherung der sprachlichen und bildenden Kunst. Dies mutet zuerst paradox an, weil wir über die Zeit sprechen, in der man die Autonomie der bildenden Kunst groß zu schreiben beginnt. Die ästhetische Emanzipation der Künste in der Tradition von Lessings Laokoon (1766), diese „eigentliche Grenzbestimmung der bildenden und redenden Künste“ (J.H. Meyer in GOETHE 1998a:106), geschah jedoch einmal selbst in sprachlichen Bildern und ist deswegen ein Ergebnis der gegenseitigen ästhetischen Bezugnahme, nicht Lostrennung der Künste ${ }^{4}$ - in dem Sinne, dass man mit Worten zu sehen, nicht bloß zu beschreiben beginnt. Noch im ausgehenden 18. Jahrhundert war es im Bewusstsein der Kunstkenner und -liebhaber nicht Lessing allein, der die neuen ästhetischen Maßstäbe setzt. J.H. Meyer notierte weiter in Literatur der Kunst und allgemeine Übersicht des Zustandes in Geschmack und Kunst vom Jahr 1750 bis 1775:

$4 \quad$ Laokoon oder über die Grenzen der Malerei und Poesie (1766) stellt die in der antiken Tradition verankerte Wesensgleichheit der Sprach- und Bildkunst in Frage und weist Malerei und Poesie ihren eigenen Darstellungs- und Zeichenbereichen zu: „Gegenstände, die nebeneinander oder deren Teile nebeneinander existieren, heißen Körper. Folglich sind Körper mit ihren sichtbaren Eigenschaften die eigentlichen Gegenstände der Malerei.

Gegenstände, die aufeinander oder deren Teile aufeinander folgen, heißen überhaupt Handlungen: Folglich sind die Handlungen der eigentliche Gegenstand der Poesie.“ (LESSING 1980:114). 
Lessing stellte in dieser Schrift, so wie ungefähr um gleiche Zeit von Mengs und Winkelmann auch geschehen war, den Grundsatz auf, daß bei der Alten Schönheit das höchste Gesetz der bildenden Künste gewesen sei, eine Maxime, welche auf Geschmack und Kunst großen Einfluß gehabt, ja man kann wohl sagen das Meiste beigetragen hat, ihnen ihre dermalige Gestalt zu geben. (J.H. Meyer in: GOETHE 1998a:106)

Winckelmanns neuen Zugang zur antiken Kunst, der nicht „ekphrastische Lobformeln“, sondern das Einzelwerk als Gegenstand und Ausgangspunkt der Reflexion ins Zentrum rückt, bezeichnet KASE (2010:207) sehr treffend als einen Übergang von der „Hermeneutik der Lektüre“ zur „Hermeneutik der Betrachtung“. Kase meint damit vor allem die Abkehr von den oben erwähnten traditionellen Formen der Kunstliteratur, bringt jedoch zugleich einen zentralen Aspekt der modernen Kunstrezeption und ebenfalls der Kunstliteratur auf den Punkt. Die zeitgenössische Kunstwissenschaft widmet sich intensiv der Sprache der Bilder in der kunstwissenschaftlichen Hermeneutik und eröffnet dazu möglicherweise neue Perspektiven auf die Kunstliteratur. Die traditionellen semiotischen und konstruktivistischen Modelle konzentrieren sich vor allem auf die Übertragbarkeit der nichtsprachlichen Expressivität des Bildes ins andere Zeichensystem und reduzieren ihr Sehspektrum auf die formal-ästhetische Ebene der jeweiligen Kunst. Aspekte der sprachlichen Kunstbetrachtung, die die Berührungspunkte zwischen Sprache und Bild in der Perspektive des Verstehens im Auge behalten, blieben oft erst einmal außer Sicht. Die Kunst des Verstehens ist nicht unbedingt auf das Medium der Sprache begrenzt, ihr aber zumeist in der geistesgeschichtlichen Tradition stark verbunden.

\section{„Je ne sais quoi“ - ein gewisses Etwas}

Wie wichtig es war, eine stimmige, adäquate Form des Sprechens und Schreibens über die Kunst zu entwickeln, die ihrer neuen Wertstellung im geistigen Interessenfeld entsprach, bezeugen die zeitgenössischen Bemühungen, diese bewusst zu fördern. Eine Rezension der kunsttheoretischen Schrift von Mengs Gedanken über die Schönheit und den Geschmack in der Malerey begrüßt sie bezeichnenderweise als einen Vorboten der wünschenswerten Entwicklung:

Die Wünsche, die wir zur Ehre und zur Beförderung der Künste in Deutschland so oft gethan, und in die nur vor kurzem ein paar verdiente Männer eingestimmt haben, daß unsere Gelehrten sich mehr in den Kunstsälen umsehen, und oft selbst die Reißfeder in die Hand nehmen, und die Künstler auch ausser ihrem Pinsel bisweilen die Schreibfeder führen möchten, diese Wünsche scheinen allmählich mehr und mehr erfüllt zu werden. (ANONYM 1763:234f.) 
Gabriela Brudzyńska-Němec

Das Interesse an der bildenden Kunst wächst in Deutschland in der zweiten Hälfte des 18. Jahrhunderts auch deswegen, weil nach 1760 mehrere Gemäldegalerien der Residenzstädte auch für nichthöfisches Publikum zugänglich gemacht wurden, die bereits berühmte königliche Sammlung in Dresden seit 1764. ${ }^{5}$ Wenn auch Dresden am Ende des Jahrhunderts sich mit seinem „größte[n] Schatz von Alterthümern“ (WINCKELMANN 1968c:224) und Gemälden als Hauptstadt der bildenden Kunst behaupten kann, bleiben die Sammlungen in Deutschland weitgehend verstreut. Die Originale bedeutender Künstler bekommt man vereinzelt und meistens für eine kurze Zeit zu Gesicht. Um den Umgang mit der Kunst harmonisch, kontinuierlich und geistig ergiebig genießen zu können, was erstrebenswert schien, griff man auch zum Schreiben über sie. ${ }^{6}$

Und dennoch verhüllt sich die Kunstbetrachtung häufig traditionell mit der Unsagbarkeitsformel „je ne sais quoi“, die sich im letzten Drittel des 17. Jahrhunderts in der Kunstliteratur etablierte. Popularisiert hat den Ausdruck „je ne sais quoi“, der auch mit der französischen, höfischen Konversationskultur in Verbindung gebracht wird, Dominique Bouhours (1628-1702) in seinem vielfach aufgelegten Buch Les Entretiens d'Artiste et d'Eugène 1671. ${ }^{7}$ Ein gewisses Etwas, was man in einem Bild ganz individuell zu bemerken glaubte, entzog sich der genauen Bestimmung und deswegen auch der eindeutigen Sprache. Diese sprachliche Verhüllung war nur teilweise ein Eingeständnis des Unvermögens, mehr aber ein Anzeichen einer neuen Sensibilität. Sie wirkte anregend, als eine Herausforderung an die Sprachkunst, das Unsägliche, das zuvor Vage dennoch anschaulich festzuhalten, auch aus einem kritischen Standpunkt heraus:

5 Oft in unmittelbarem Zusammenhang mit den Akademiegründungen, so in Dresden (1764), Wien (1770) und Düsseldorf (1771), sowie der Reorganisation der Berliner Akademie (1786). Vgl. Penzel (2007:51).

$6 \quad$ Es entstehen besonders viele Beschreibungstexte, publiziert meistens in den Zeitschriften zwischen 1780 und 1800. Nach KASE (2010:19-21) entwickelt sich in Deutschland die von den Beschreibungen dominierte Kunstliteratur intensiver als im zentralisierten Frankreich, wo die Sammlungen auch schwerer zugänglich sind.

7 Vgl.: „Das überraschende und verzaubernde ,je ne sais quoi` ist hier so wichtig, weil es in der Rezeptionsästhetik eine erste nachdrückliche Verschiebung des ästhetischen Urteils auslöst: Es verschiebt sich von der regelgeleiteten Werkkritik zur erfahrungsgelenkten Selbstwahrnehmung und präfiguriert damit die Kategorie des ,Sublimen“.“ (KASE 2010:63) 
„Was halten Sie von den Menschen“, sagte einst Guibal zu mir (Mengs), „die sich Kenner zu seyn dünken, und über ein Gemälde von berühmten Meistern Alles gesagt zu haben glauben, wenn sie nach einer flüchtigen Betrachtung, mit einem Anschein von Begeisterung ausrufen: ,Ach wie schön! oh, unnachahmlich! Man gewahrt in den Werken dieses Meisters ein gewisses je ne sais quoi, das bezaubert, aber durchaus nicht beschrieben und noch weniger nachgeahmt werden kann.““ (MENGs 1843:251)

Mengs’ Position war klar, „ein gewisses je ne sais quoi“ lässt sich erklären und folglich auch beschreiben. Deswegen ermutigte er seine Schüler in der oben zitierten Rede, dem ,je ne sais quoi“ auf den Grund zu gehen, und keineswegs vor großen Kunstwerken in stumme Begeisterung und künstlerische Untätigkeit zu verfallen. „Gewahrt man bei einem Werke einen Theil, der das Herz, ohne dass man sich Rechenschaft darüber zu geben vermag, bewegt und unsere Empfindung rege macht, so muss man genau beobachten [...].“ (MENGS 1843:253)

Mengs' Empfehlung einer methodischen, kunstgerechten Untersuchung des Gemäldes als Antwort auf das ,je ne sais quoi“ legt Grundlagen für eine neue Form der Kunstbetrachtung, die neben fachmännischer Rubrikenbeschreibung oder dilettantischem Enthusiasmus ein Kunstverständnis auf der Basis des Gefühls fördert, als sinnlich bedingte und zugleich reflektierte Erfahrung gedacht. So plädierte Mengs auch für die Beschreibungen der Kunstwerke, auch als eine Form der pädagogischen Vermittlung des Kunstverständnisses, weit effektiver als herkömmliche Künstlerviten. ${ }^{8}$

\section{Schönheit reden}

„Man sehe sie an“, heißt es in den ersten Sätzen von Winckelmanns Beschreibung des Torso im Belvedere zu Rom,

[...] als eine Probe von dem, was über ein so vollkommenes Werk der Kunst zu denken und zu sagen wäre, und als eine Anzeige von Untersuchung in der Kunst. Denn es ist nicht genug zu sagen, daß etwas schön ist: man soll auch wissen, in welchem Grade, und warum es schön sey. (WINCKELMANN 1968b:169) ${ }^{9}$

8 Erstmals zusammen mit Winckelmann in einem Brief an Johann Georg Wille 1756. „Die geforderte Beschreibung von Kunstwerken soll die Realisierung gestalterischer Grundsätze veranschaulichen und die Gründe ihrer Schönheiten aufzeigen.“ (KASE 2010:208).

$9 \quad$ Beschreibung des Torso im Belvedere zu Rom wurde zuerst 1759 in der Bibliothek der schönen Wissenschaften und freyen Künste veröffentlicht. 
Die Beschreibung des Torso, wie damals angenommen, einer HerkulesStatue „in Absicht des Ideals“ (WINCKELMANN 1968b:169), kündigt ein neues Bestreben an: „eine Probe von dem, was [...] zu denken und zu sagen wäre“. ${ }^{10}$ Durch die Beschreibung als einen in erster Linie kommunikativen, auf keine Vollständigkeit zielenden Versuch lernt man das Schöne zu verstehen. Das Schreiben wird nicht lediglich zum Festhalten der Tatsachen genutzt. Die Beschreibung gestaltet das Sehen, wird zum Ausdruck eines verstehenden Blickes. ${ }^{11}$ Die „Hermeneutik der Betrachtung“ (KASE 2010:207), die das Verstehen der Texte im Umgang mit der Kunst zuerst ersetzt, bewahrt einen lektürebezogenen Kontext. Die Wendung zu der sinnlichen aufmerksamen Anschauung und Abkehr von bloßer Schriftgelehrsamkeit hängt mit der Bindung des kunstgerechten Sehens an die sprachliche Empfindlichkeit des Schönen zusammen. Sie kommt nicht zuletzt aus der Lektüre heraus. Den Gedancken über die Nachahmung der Griechischen Werke in der Mahlerey und Bildhauer-Kunst stellt Winckelmann die berühmten Worte voran:

Der einzige Weg für uns, groß, ja, wenn es möglich ist, unnachahmlich zu werden, ist die Nachahmung der Alten, und was jemand vom Homer gesagt, daß derjenige ihn bewundern lernet, der ihn wohl verstehen gelernet, gilt auch von den Kunst-Wercken der Alten, sonderlich der Griechen. Man muß mit ihnen, wie mit seinem Freunde, bekannt geworden seyn, um den Laokoon ebenso unnachahmlich als den Homer zu finden. (WINCKELMANN 1968:30)

Winckelmanns Kunstschriften, vor allem die Beschreibungen, bezieht man oft auf seine ursprünglichen künstlerischen Anlagen, deren Verwirklichung ihm versagt war. Der Künstlerblick bewirkte in ihnen eine äußerste Stilbewusstheit, „die in der Beschreibung der Kunstwerke, in der Kunstbetrachtung, im Prozeß der Vermittlung das leisten muß, was dem dilettierenden

10 Winckelmann teilt seine Beschreibungen grundsätzlich in zwei Arten nach dem Ideal und nach der Kunst. Nach TraUTwEIN (1997:166): „In einem Brief wird er genauer, teilt ein in die Darstellung einer idealen und poetischen Idee in gehobenem Stil, in eine Beschreibung von allem, was die Kunst an Erfahrbarem bietet und eine Abhandlung der Gelehrsamkeit. Eine Systematik der Beschreibung jedoch fehlt. Da wird klar, daß das Beschreibungsproblem auch ein Problem seiner ganzen Forschung ist.“

11 Vgl. Pfotenhauer (1995:326) über die Beschreibung des Torso: „Winckelmann gerät hier nachgerade in ein hermeneutisches Delir. Nicht deshalb sei dies behauptet, weil er den Torso als den eines Herakles deutet [...]. Vielmehr lässt Winckelmann den ruhenden, vor der Apotheose stehenden Helden wie folgt auf seine Taten zurückblicken und bemüht dabei förmlich Kaskaden von Mythologemen und Auslegungsaspekten.“ 
Künstler Winckelmann für immer versagt bleiben wird“ (TRAUTWEIN 1997: 87f.). Winckelmann bezieht sich in den oben zitierten Worten dazu auf eine literarisch geschulte Sensibilität, die ihren Ursprung in Lektüre und im Verstehen literarischer Texte hat. Sie regt die sinnlich-anschauliche Empfindlichkeit nicht weniger an als der künstlerische Kennerblick. Auf die Schlüsselrolle der Lektüre, als einer Fähigkeit, die Feinheiten des Textes wahrzunehmen und sie nachzufühlen, verweist Winckelmann mehr als einmal. Erst „wenn in Lesung eines Scribenten die Empfindung zärtlich gerühret wird“ (WINCKELMANN 1968c: 216), wird eine Voraussetzung zur Empfindung des Schönen der bildenden Kunst geschaffen. Die Ausbildung eines jungen Menschen zum guten Geschmack in der Kunst beginnt beim Lesen:

Zuerst sollte dessen Herz und Empfindung, durch Erklärung der schönsten Stellen alter und neuer Scribenten, sonderlich der Dichter, rührend erwecket, und zu eigener Betrachtung des Schönen in aller Art zubereitet werden, weil dieser Weg zur Vollkommenheit führet. (WINCKELMANN 1968c:221)

„Wo die Empfindung nicht ist, predigt man Blinden die Kenntniß des Schönen, wie die Musik einem nichtmusikalischen Gehöre.“ (WINCKELMANN 1968c:216) Der Gedanke geht womöglich doch über das Wachrufen einer allgemeinen ästhetischen Sensibilität hinaus. Die Lektüre wird zur Schule der genauen Anschauung. In Erinnerung über die Betrachtung der Werke der Kunst, einem Text, ähnlich wie Beschreibung des Torso, aus dem Jahre 1759, veranschaulicht Winckelmann didaktisch den stilistischen Unterschied zwischen einem guten und einem schlechten Kunstwerk. ${ }^{12}$ Eine Vergleichsfläche bietet ihm dazu die stilistische Abfassung eines Buches:

Ein sehr mühsam gemachtes Bild vom Mahler oder Bildhauer ist, bloß als dieses, mit einem mühsam gearbeiteten Buche zu vergleichen. Denn so wie gelehrt zu schreiben nicht die größte Kunst ist, so ist ein sehr fein und glatt ausgepinseltes Bild allein kein Beweis von einem großen Künstler. Was die ohne Noth gehäuften Stellen vielmals nie gelesener Bücher in einer Schrift sind, das ist in einem Bilde die Andeutung aller Kleinigkeiten. [...]

Es können Bücher und Werke der Kunst gemacht werden, ohne viel zu denken; ich schließe von dem, was wirklich ist: ein Mahler kann auf diese mechanische Art eine Madonna bilden, die sich sehen läßt, und ein Professor so gar eine Metaphysik schreiben, die tausend jungen Leuten gefällt. Die Fähigkeit des Künst-

12 Veröffentlicht ebenfalls, wie die Beschreibung des Torso, in der Bibliothek der schönen Wissenschaften und freyen Künste. Im Rahmen einer kurzen, aber ergiebigen Zusammenarbeit mit dem Leipziger Blatt erschienen 1759 fünf Aufsätze von Winckelmann. 
Gabriela Brudzyńska-Němec

lers zu denken aber kann sich nur in oft wiederholten Vorstellungen, so wie in eigenen Erfindungen zeigen. [...]

Denn wie es schwerer ist, viel mit wenigem anzuzeigen, als es das Gegentheil ist, und der richtige Verstand mit wenigem mehr als mit vielem zu wirken liebet; so wird eine einzelne Figur der Schauplatz aller Kunst eines Meisters seyn können. Aber es würde den mehresten Künstlern ein eben so hartes Gebot seyn, eine Begebenheit in einer einzigen oder in ein paar Figuren, und dieses in groß gezeichnet vorzustellen, als es einem Scribenten seyn würde, zum Versuch eine ganz kurze Schrifft aus eigenem Stoff abzufassen: denn hier kann beider Blöße erscheinen, die sich in der Vielheit verstecket. (WINCKELMANN 1968a:149f.)

Die ganze Reihe von Text-Bild-Analogien lässt sich mit dem an sich schon problematischen Begriff ,Bilder lesen“ nur vage beschreiben. ${ }^{13}$ Winckelmann spielt in seinen Vergleichsbeispielen auf keine direkte Kommunikationssituation an, eine sich im Rahmen des Verstehensaktes entwickelnde, sprachlich fassbare Dialogizität zwischen dem Bild und dem Rezipienten. Winckelmanns Modell der Bildanalyse, Bildlektüre ist zuerst an eine rhetorische Textstruktur angelehnt. Er vergleicht unmittelbar die formalen Gestaltungselemente der bildlichen und sprachlichen Ausdrucksweise, von der Art der stilistischen Feder- und Pinselführung bis zu Kompositionselementen und rhetorischen Mitteln. Eine Textanalyse wird auf das Bild übertragen, eine Bildanalyse auf den Text. ${ }^{14}$

Es handelt sich jedoch dabei um kein Übersetzungsverfahren, bei dem unterschiedliche, jedem Medium eigene Zeichensysteme ineinander übertragen und so verständlich gemacht werden. Winckelmann spürt vielmehr den in der Rhetorik verankerten Formen und Verfahren nach, die vor allem in ihrer Wirkung auf den Hörer und Leser charakteristisch sind. So kommen nicht unbedingt neue formale Verfahren ans Licht. Alle oben genannten bildlichen Gestaltungselemente lassen sich auch ohne Bezugnahme auf den Text festhalten und analysieren. Die „Andeutungen aller Kleinigkeiten“ in einer Sta-

13 Vgl. die Kritik von Kase (2010:81): „,,Bilder lesen“ ist in der Kunstgeschichte eine verbreitete, aber meist sehr unreflektiert verwendete Formel für eine gelingende Bildbetrachtung, die stillschweigend eine vage Lesehermeneutik auf die Bildhermeneutik überträgt und damit eine Analogie des Verstehens von Text und Bild suggeriert.“

14 Erst bei OsKar WALZEL (1917) wird dieser Gedanke in der Wechselseitigen Erhellung der Künste weiter entwickelt, indem er dafür plädiert, die Kategorien der Bildbetrachtung, z.B. Lineares und Malerisches, Flächenhaftes und Tiefenhaftes, und nicht zuletzt Vielheit und Einheit, die wir auch bei Winckelmann treffen, für die Deutung der Poesie fruchtbar zu machen. 
tue von Bernini werden dennoch erst augenfällig in der Parallele zu den „ohne Noth gehäuften Stellen vielmals nie gelesener Bücher“ (WINCKELMANN 1968a:149) - eben nicht als Anzeichen der Schönheit und künstlerischen Vollkommenheit. Die fehlende Originalität wird deutlich im geistigen Vorwurf. Erst wenn man nach der Bedeutung und Wirkung des Ganzen fragt, kann man richtig unterscheiden, deswegen die noch weiter führende Schlussfolgerung Winckelmanns: „Eben so sind keine Kennzeichen, an welchen der Fleiß allein Antheil hat, fähig zur Kenntniß oder zum Unterschiede des Alten vom Neuen.“ (WinCKELMANN 1968a:149) Die Aussage ist eindeutig: Eine auf rein formale bildliche Ausführung konzentrierte Analyse des Kunstwerks, wenn auch eine sorgfältige, reicht nicht aus, um es richtig zu erläutern, im Sinne Winckelmanns , die Alten zu verstehen'. Das Zurückgreifen auf die Textdeutung stellt dagegen Auslegungsmodelle bereit, die sich die Freiheit nehmen, das Kunstwerk vor allem in seiner Wirkung auf den Betrachter zu sehen, denn wie Winckelmann es in der Abhandlung von der Fähigkeit der Empfindung des Schönen formuliert: „Unsere Betrachtung sollte anheben von den Wirkungen des Verstandes, als dem würdigsten Theile, auch der Schönheit, und von da heruntergehen auf die Ausführung." (WINCKELMANN 1968c:232) Und dennoch, es ist bei weitem keine nur der begrifflichen Rezeption verpflichtete Optik, sondern eine, die das Gesehene, sinnlich Wahrgenommene, auf den Betrachter wirken lässt. So ist die Schönheit - „diese geht eigentlich die Bildung an, und ist die höchste Absicht der Kunst“ - in einem rhetorischen Sinne, als Exempel, ein Zugang zum Schönen, ,jenes erstreckt sich auf alles, was gedacht, entworfen und ausgearbeitet wird“ (WINCKELMANN 1968c:213).

Gert Ueding, der auf eine enge Verbindung des Winckelmann'schen Kunstdenkens mit der Tradition der Rhetorik hinweist, bemerkt hier den Punkt, „an dem platonische Metaphysik und pragmatische rhetorische Kunsttheorie zusammentreffen können (also nicht etwa die eine die andere ablöst) und aus ihrer Verbindung die neuzeitliche Ästhetik entsteht.“ (UEDING 1995:49) ${ }^{15} \mathrm{Er}$ referiert zumeist allgemein die der Rhetorik als Verfahren mehr als der Dialektik zugewandten philosophischen Grundlagen der Kunstauffassung Winckelmanns, indem er auch auf seine kritische Position hinweist (UEDING

15 Vgl. auch Ueding (1995:45): „Die Fundierung der neuzeitlichen Kunst auf die Nachahmung der Alten bedeutet also die Übertragung einer dem Kanon der exempla verpflichteten Literaturauffassung auf die Werke der bildenden Kunst. [...] und Winckelmann beruft sich zur Rechtfertigung seines Vorgehens auch ständig auf das literarisch-poetologisch längst legitimierte Paradigma.“ 
Gabriela Brudzyńska-Němec

1995:53). Wie dicht Winckelmanns praktische Kunstbetrachtung in die Schreib- und Lesekunst verflochten ist, macht eine weitere Gegenüberstellung zweier Kunstwerke erkennbar, die er wiederholt heranzieht, Homers Werke und Laokoon:

Im Laocoon siehst du bey dem Schmerz den Unmuth, wie über ein unwürdiges Leiden in dem Krausen der Nase, und das väterliche Mitleiden auf den Augapfeln wie eine trübe Duft schwimmen. Diese Schönheiten in einem einzigen Drucke sind wie ein Bild in einem Worte beym Homerus, nur der kann sie finden, welcher sie kennt. Glaube gewiß, daß der alten Künstler so wie ihrer Weisen Absicht war, mit wenigem viel anzudeuten: Daher lieget der Verstand der Alten tief in ihren Werken [...].

Ist ein Vorurtheil nützlich, so ist es die Ueberzeugung von dem, was ich sage; mit derselben nähere dich zu den Werken des Alterthums, in Hoffnung viel zu finden, so wirst du viel suchen. Aber du musst dieselbe mit großer Ruhe betrachten; denn das viele im wenigen und die stille Einfalt wird dich sonst unerbauet lassen, wie die eilfertige Lesung des ungeschmückten großen Xenophon. (WINCKELMANN 1968a:150f.)

Das Finden des Schönen setzt die Kenntnis der Schönheit voraus, die sich in den wiedererkennbaren formalen Mitteln ausdrückt, sichtbar gemacht wird. In der obigen Beschreibung von Laokoon verweist Winckelmann dabei erneut auf eine literarische Form, die Figur der bildlichen Charakterisierung der körperlichen Gegenstände, wie man sie in Homers Werk findet: mit einer einzigen Eigenschaft, „in einem einzigen Drucke“. Das literarische Verfahren hat später Lessing im Laokoon als Beispiel einer malerischen Sprache gedeutet, die auf die Vielheit verzichtet, um anschaulich zu wirken:

Ebenso kann auch die Poesie in ihren fortschreitenden Nachahmungen nur eine einzige Eigenschaft der Körper nutzen, und muß daher diejenige wählen welche das sinnlichste Bild des Körpers von der Seite erwecket, von welcher sie ihn braucht.

[...]

Für ein Ding, sage ich, hat Homer gemeiniglich nur einen Zug. Ein Schiff ist ihm bald das schwarze Schiff, bald das hohle Schiff, bald das schnelle Schiff, höchstens das wohlberuderte schwarze Schiff. Weiter lässt er sich in die Malerei des Schiffes nicht ein. (Lessing 1980:115f.)

Den rhetorischen Kunstgriff, der auf eine bildliche Wirkung zielt, kehrt Winckelmann in ein Rezeptionsverfahren um. Auf diese Weise gewinnt auch seine berühmte Formel von der „stillen Einfalt“ einen neuen Deutungsaspekt, nicht nur als ein Harmonie- und Stimmigkeitsgebot, sondern ebenfalls als rezeptionsästhetische Forderung - das „viele im wenigen“ zu suchen (WINCKELMANN 1968a:151). Es ist eine Deutungs- und Verstehensforderung, die aus dem Wirkungsmuster der Literatur entfaltet wird. Daher gründet die 
Schönheits-Definition in der Erinnerung über die Betrachtung der Werke der Kunst ebenfalls auf einer Vorstellung von der gestaltenden, bildenden Leistung der Rede:

Der höchste Vorwurf der Kunst für denkende Menschen ist der Mensch, oder nur dessen äußere Fläche, und diese ist für den Künstler so schwer auszuforschen, wie von den Weisen das Innere desselben, und das schwerste ist, was es nicht scheinet, die Schönheit, weil sie eigentlich zu reden, nicht unter Zahl und Maaß fällt. [...] und wenn auch das Schöne durch einen allgemeinen Begriff könnte bestimmet werden, welches man wünschet und suchet, so würde sie dem, welchem der Himmel das Gefühl versaget hat, nicht helfen. (WINCKELMANN 1968a:151f.)

Winckelmann will die äußere Schönheit des Menschen, die höchste der Kunst, weder einer mathematischen Proportionslehre noch einem philosophischen Begriff unterordnen. Die Formel „Schönheit reden“ ist im Kontext seiner Schrift kein beliebiger Ausdruck. Dem Sinn dieser Worte folgend, wäre eine Fixierung der Schönheit, dadurch auch ihre Bestimmung, erstmals in der Rede über sie denkbar, einer Rede, die den Begriff und das Gefühl vereinigt. Die Rolle der Sprache im Kunstverstehen wird dadurch sehr aufgewertet. Nicht die schriftliche Überlieferung steht im Vordergrund, sondern die Lektüre als Gefühlslehre und die Redekunst als Entfaltung des Fühl- und Verständnisvermögens.

\section{„Nimm meine Augen“}

Auch in der heutigen kunstgeschichtlichen Hermeneutik wendet man sich mit großem Interesse der Rede zu. Die Zusammenhänge zwischen Bild und Text im Gemälde, dem eine schriftliche Geschichte zur Vorlage dient, erörtert Oskar Bätschmann aus der Sprechakt-Theorie von Austin und Searle heraus und erkennt eine grundsätzliche Nähe des Bildes zur Unmittelbarkeit der Redesituation, die sich in einer konkreten, einmaligen Darstellung der Affekte im Bild wiederfindet: „Indem das Bild die Situation der Rede wiederherstellt, erlangt es die Ähnlichkeit mit dem Ereignis, die der Text aufgegeben oder verloren hat.“ BÄTSCHMANN (2009:148) bezeichnet dies als eine „vergangene Gegenwärtigkeit“. ${ }^{16}$

16 Vgl. weiter BätschmanN (2009:148): „Mit der Darstellung der Affekte im Bild werden zum Text die illokutionalen Akte erfunden, also diejenigen Akte, die nicht den Text, sondern die Rede charakterisieren. Man kann daher sagen, ein Gemälde rekonstruiere durch den Text hindurch die Situation der Rede, indem 
Die bildtheoretischen Reflexionen der letzten Jahrzehnte konzentrieren sich auf die besondere „Leistung des Bildes“, auf dessen Potenzial, auch sprachlos zum Auge zu sprechen (BOEHM 2005:30). Das hermeneutische, dynamische Verständnis des Bildes als eines „Seinsvorgangs“, das dem Kunstwerk „die Eingriffe, Einsichten und Deutungen“ (BOEHM 2007:254) in die bzw. der Wirklichkeit zubilligt, hat eine theoretische Nähe zu grundlegenden Bildreflexionen des 18. Jahrhunderts, auf die näher eingegangen werden soll. Aus der oben erwähnten, von Winckelmann geforderten genauen Betrachtung des Kunstwerks, aus dem Umgang mit ihm „wie mit seinem Freund“ (WINCKELMANN 1968:30), ergibt sich eine kommunikative Situation, aber wiederum nicht zwischen dem Bild und dem Betrachter, sondern zwischen dem, der sieht, und demjenigen, dem das Sehen vermittelt werden soll. „In solcher genauen Bekanntschaft wird man wie Nicomachus von der Helena des Zeuxis urtheilen: ,Nimm meine Augen', sagte er zu einem Unwissenden, der das Bild tadeln wollte, ,so wird sie dir eine Göttin scheinen'“"(WINCKELMANN 1968:30)

Winckelmann legt diese Worte dem antiken griechischen Maler Nikomachos in den Mund, der Zeuxis' Darstellung der Helena betrachtet. Ein fachkundiger Blick wird hier gefordert, der weiterzugeben ist. Der Sehende steht dabei im Mittelpunkt und seine Sprache wird zur Sprache der Kunst stilisiert, ja fast mit ihr identisch gesetzt; nicht im Sinne einer medialen Bild-WortÜbersetzung, auch nicht ausschließlich einer sinnlichen Einfühlung, sondern einer erfahrenen Identität. Man könnte von einer „ästhetischen Nichtunterscheidung ${ }^{17}$ sprechen, die nicht die Darstellung und das Dargestellte, sondern das Kunstwerk und seinen Betrachter betrifft, das „Enthaltene“ und das „Enthaltende“, wie Winckelmann es formuliert:

Die Fähigkeit das Schöne in der Kunst zu empfinden, ist ein Begriff, welcher zugleich die Person und Sache, das Enthaltende und das Enthaltene in sich fasset, welches ich aber in eins schließe, so daß ich hier vornehmlich auf das erstere mein Absehen richte [...]. (WINCKELMANN 1968c:212)

Aus dieser Wirkungs- und Rezeptionsästhetik in einem erwächst eine Sprache des Sehenden, die eine besondere Nähe zu dem betrachteten Gegenstand

es mit der Erfindung und Darstellung der Bewegungen und Affekte als illokutionaler Akte die Verbindung mit dem Ereignis gewinnt, von dem der Text ausgegangen ist.“

17 Gadamers Kategorie der ästhetischen Nichtunterscheidung „umschreibt eine Erfahrung, bei der die Sache und ihre sich darstellende Präsenz völlig miteinander verschmelzen“ (BoEHM 2005:30). 
aufbaut, nicht auf der Basis der stark individualisierten Einfühlung, sondern einer durchaus kommunizierbaren Identifikation, die auf dem Verstehen des Kunstwerks beruht und durchaus redefreudig ist. Die eindeutige Wende zum Betrachter hin, die Winckelmann vorschlägt, zum „Enthaltenden“, der Person, die die Sache, das „Enthaltene“, in sich aufnimmt, steht jenseits der Diskussion über das objektive oder subjektive Verständnis des Schönen, sie stellt vor allem die vielschichtigen Kommunikationsprozesse in den Vordergrund. ${ }^{18}$ „Mitteilung und Empfänglichkeit sei übrigens das Losungswort“, pointiert auch GOETHE (1998:692) das Kunstinteresse seiner Zeit in seiner theoretisch-literarischen Schrift Der Sammler und die Seinigen 1799 (vgl. BRUDZYŃSKA-NĚMEC 2012).

\section{„Das wahre Gefühl des Schönen“}

Dass die Schönheit, als ausschlaggebende ästhetische Kategorie, auf die Kunst verpflichtet wird, gehört zum klassizistischen Ideal (vgl. LIESSMANN 2009:51-67). Es ist bezeichnend, dass sowohl Winckelmann als auch Mengs die Schönheit nicht allgemein als einen abstrakten Begriff, sondern sie vor allem in ihrer Wirkung betrachten, als „das wahre Gefühl des Schönen“. Die Bildung und die Voraussetzungen des Gefühls des Schönen, für die Winckelmann immer neue sprachliche Bilder findet, zeichnen seine praktisch orientierte Kunsttheorie vor, ja, sie geben ihr die Kontur:

Das wahre Gefühl des Schönen gleicht einem flüssigen Gipse, welcher über den Kopf des Apollo gegossen wird, und denselben in allen Theilen berühret und umgiebt. Der Vorwurf dieses Gefühls ist nicht, was Trieb, Freundschaft und Gefälligkeit anpreißen, sondern was der innere feinere Sinn, welcher von allen Absichten geläutert seyn soll, um des Schönen willen selbst, empfindet. (WiNCKELMANN 1968c:217)

18 Vgl. Tatarkiewicz (2003:310-316) über die Ästhetik der Aufklärung. KaRL PhILIPP MoRITZ (1962:101), der als Verfechter einer poetischen Sprache der Kunstbetrachtung und deswegen als ein kunsttheoretischer Antipode Winckelmanns dargestellt wird, geht von demselben Standpunkt des Sehenden in seinen Reflexionen aus, doch mit anderen Konsequenzen für die Kunstbeschreibung. „Daher kömmt nichts unter allem Sichtbaren dem Sehenden selbst an Schönheit gleich, und die sanfte Spur des Sehenden, in seine ganze Umgebung verhältnismäßig eingedrückt, ist von allem Sichtbaren allein vermögend, uns unmittelbar Liebe und Zärtlichkeit einzuflößen.“ 
Die Gefühle, von denen hier die Rede ist, sind nicht sentimental, sondern dynamisch, anregend, formend. Nach Zedlers Lexikon spricht man von der „Eindruckung, Impressio“, in Bezug auf seelische Vorgänge, „wenn man sich etwas feste einbildet, und dem Gedächtniß gleichsam einprägt“ (ZEDLER 1732-1754:541). Die Eindrücke sind in diesem Verständnis nichts Flüchtiges oder Vergängliches, sondern sie gestalten die Wahrnehmung und prägen die innere Disposition des Menschen. Diese klassische Lehre der auf Psychologie zurückgreifenden, „subjektivistischen Ästhetik“ (TATARKIEWICZ 2003:310) wird durch Winckelmann einer konstruktiven Kritik unterzogen, oder besser gesagt, verfeinert: Er schreibt, dass „die ersten Eindrücke die stärksten sind, und vor der Ueberlegung vorhergehen: was wir durch diese empfinden, ist schwächer“ (WINCKELMANN 1968c:219). Dass durch einen starken Eindruck eine schwache Empfindung entsteht, wirkt zuerst paradox. Winckelmann erklärt diese Diskrepanz, indem er eine Schlüsselfunktion in der Vermittlung entdeckt:

Alle heftige Empfindungen gehen über das Mittelbare hinweg zum Unmittelbaren, da das Gefühl hingegen gerühret werden soll, wie ein schöner Tag entstehet, durch Anmeldung einer lieblichen Morgenröthe. Es ist auch die heftige Empfindung der Betrachtung und dem Genusse des Schönen nachtheilig, weil sie zu kurz ist: denn sie führet auf einmal dahin, was sie stuffenweise fühlen sollte. Auch in dieser Betrachtung scheint das Alterthum ihre Gedanken in Bilder eingekleidet zu haben, und verdeckte den Sinn derselben, um dem Verstande das Vergnügen zu gönnen, mittelbar dahin zu gelangen. (WINCKELMANN 1968c:219)

Das „Unmittelbare“ und „Mittelbare“ sind die Wesensmerkmale der Wahrnehmung, auf die sich Winckelmann konzentriert und die er ästhetisch neu auswertet. Die unmittelbare sinnliche Wirkung der bildenden Kunst, der althergebrachte Topos, der ihren Vorteil gegenüber der Poesie beschreibt, wird einem mittelbaren Effekt unterstellt, einer durchaus sprachlichen Wirksamkeit. Das durch den äußeren Sinn erkannte, anschauliche „auf einmal“ des Bildes bedarf einer sukzessiven, stufenweisen Vermittlung des inneren Sinnes. Sie garantiert nicht nur das Verstehen, sondern vor allem die fortdauernde Empfindung des Schönen. ${ }^{19}$ Das klassizistische Modell der edlen Einfalt, der idealisierten Schönheit, enthüllt sich in diesem Licht wiederum als eine Form der ,Ver-dichtung', die einer Auslegung bedarf. ${ }^{20}$

19 Vgl. WincKelmanN (1968c:217): „Das Werkzeug dieser Empfindung ist der äußere Sinn, und der Sitz derselben der innere: jener muß richtig, und dieser empfindlich und fein seyn.“

20 In der Kunsttheorie des 20. Jahrhunderts war es vor allem Erwin Panofsky, der auf das Phänomen des Mittelbaren in der bildenden Kunst eingegangen ist. In- 
Wenn der äußere Sinn richtig ist, so ist zu wünschen, daß der innere diesem gemäß vollkommen sey: denn es ist derselbe wie ein zweyter Spiegel, in welchem wir das Wesentliche unserer eigenen Aehnlichkeit, durch das Profil, sehen. Der innere Sinn ist die Vorstellung und Bildung der Eindrücke in dem äußeren Sinne, und, mit einem Worte, was wir Empfindung nennen. (WINCKELMANN 1968c:218)

Die Metapher des zweiten Spiegels, die die eigentliche Kunstempfindung veranschaulichen sollte, ist auch für den Charakter der Kunstliteratur zutreffend. Winckelmann entwickelt eine Lesart des Kunstwerks und bedient sich dabei vor allem der Sprache, die durch ihre Verbindung mit der Anschauung, eben als Werkzeug des inneren Sinnes und durch ihre rhetorische Ausdruckskraft, fähig ist, den Eindruck in eine Empfindung zu verwandeln und ihr letztendlich Gestalt zu geben. Diese Art der Kunstliteratur, die auf sprachliche Ausformung der Schönheit gerichtet ist, fördert nicht nur eine Präzision der Betrachtung, sie entwickelt auch eine formale Nähe zu ihrem Gegenstand, der bildenden Kunst. Sie ist ebenfalls keine sprachliche Abbildung, sondern eine sprachliche Vermittlung, die selbst die Gestalt einer rhetorischen Kontur annimmt, die „berühret und umgiebt“ (WINCKELMANN 1968c:217). Das Schreiben und Reden über die Kunst im Allgemeinen fasst Winckelmann nach einem ähnlichen Prinzip, als eine Gestaltungskunst, die über die begriffliche Klarstellung hinausführt und eine Deutungs- und Verstehensleistung des Lesers voraussetzt. Seine Geschichte der Kunst als „Lehrgebäude“ “21 kann man auch in diesem Sinne verstehen, als eine klare gedankliche Struktur, in der man sich dennoch frei bewegen kann und muss bzw. sollte.

dem Panofsky sich auf Heideggers „Gewalt der Interpretation“ als Auslegung philosophischer Schriften stützt, die darauf beruht, nicht das zu zeigen, was im Text „ausdrücklich“ gesagt wurde, sondern „was noch Ungesagtes durch das Gesagte vor Augen“ gelegt wird, zieht er die Konsequenz für die Bildbeschreibungen seines Faches: „Auch sie, sogar das scheinbar unproblematische Aufzeigen eines bloßen Phänomensinns, legen im Grunde ,Ungesagtes vor Augen“.“ (PANOFSKY 1992:217)

21 Vgl. WincKelmann (1968d:235): „Ich nehme das Wort Geschichte in der weiteren Bedeutung, welche dasselbe in der Griechischen Sprache hat, und meine Absicht ist, einen Versuch eines Lehrgebäudes zu liefern.“ 
Gabriela Brudzyńska-Němec

\section{Was wir Schönheit nennen}

Die Art, theoretisch über Kunst zu schreiben, die auf begriffliche Schärfe zugunsten eines neuen bildhaften Stils verzichtet, traf vor allem in Kreisen der philosophisch geschulten Kunstkenner auf Vorbehalte. Erfahren hat dies ausdrücklich Anton Raphael Mengs. Die Schrift Gedanken über die Schönheit und den Geschmack in der Malerey veröffentlichte er anonym bei dem Züricher Maler, Buchhändler und Kunstschriftsteller Johann Caspar Füssli (1706-1782), mit Winckelmanns engagierter Unterstützung. Die kritischen Diskussionen sind in die Vorrede des Verlegers eingegangen. In großen Teilen trägt sie eine Rechtfertigung des für eine kunsttheoretische Schrift ungewöhnlichen Stils vor:

Denn der Verfasser schrieb nicht Auslegungsweise, weil er sich ein großes Werk, dem Leser nicht das Nachdenken ersparen wollen. Es war auch überflüssig die Wahrheit, die itzt in wenig Blättern ist, in Bände zu streuen, wer ein Talent zur Kunst hat, müßte was er hier in ununterbrochener Reyhe antrift, dort, nur mühsam suchen, der aber hier nichts findet, würden keine Folianten erleuchten. So viel von dem Wesentlichen der Schrift. Der Ausdruck, die Sprache, sind nicht blumigt, aber kräftig, aber dem Lehrer der ungeschmückten Wahrheit gemäß, und kleiden den Gedanken, wie an den Bildsäulen der alten das leichte Gewand Götter und Helden kleidet, geworfen den Held den es umgiebet mit Anstand zu zeigen, nicht verstecken. (Füssli in MENGs 1774:6f.)

Die Vorrede von Füssli hebt das Essenzielle der Schreibart von Mengs hervor, ihren verhüllenden, andeutenden und zugleich einheitlichen (ununterbrochenen) Charakter. Die Sprache zeichnet das Wesentliche des Gedankens nach, wie ein Faltenwurf eine Statue. Diese plastische, wiederum metaphorische Formel, die die Literatur der Kunst mit einem die Gedanken einhüllenden Gewand gleichsetzt, erinnert an Winckelmanns Abguss-Metapher, die „in allen Theilen berühret und umgiebt“ (WINCKELMANN 1968c:217). In diesem Sinne wird das Wesen der Schönheit von Mengs nicht definiert, sondern mit einem metaphorischen Bild gezeichnet. Dennoch wird das metaphorisch-allegorische Gleichnis angewendet, um zu definieren, was eigentlich der Stein des Anstoßes war und auf die Veränderungen der Kunstliteratur hindeutete. Das Bild veranschaulicht den abstrakten Begriff, indem seine Attribute als Attribut der Schönheit sichtbar werden. Schönheit, die Mengs in der platonischen Tradition mit dem Begriff der Vollkommenheit in Verbindung bringt, sei so zu verstehen wie ,der mathematische oder unzertheiliche Punkt“ (Mengs 1774:18).

Da die Vollkommenheit mit der Menschlichkeit nicht übereinstimmen kann, und allein bey Gott ist, von dem Menschen aber nichts wirklich begriffen wird, als 
was unter die Sinne fällt; so hat ihm Allweise einen sichtlichen Begriff der Vollkommenheit eingeprägt, und dieses ist, was wir Schönheit nennen. Also sage ich: Sie ist in allen erschaffenen Dingen, nämlich wenn der Begriff so wir von den Dingen haben, und unser intellectuales Gefühl nicht höher mehr in der Einbildung gehen kann, als wir die Materie sehen: Dieses ist zu vergleichen mit der Natur des Punktes; ein Punkt soll unzertheilich seyn, also ist der Punkt in der Wahrheit allezeit unbegreiflich: Weil wir aber nöthig haben uns einen sichtlichen Begriff des Punktes zu machen, so heissen wir einen Punkt denjenigen Flek in welchem wir die Zertheilung nicht mehr würken können: Diesen heißt man den sichtlichen Punkt. (Mengs 1774:17f.)

Die sichtbare Ganzheit und Untrennbarkeit des Punktes stehen für Kumulation und Unvollkommenheit zugleich. Die „Schönheit“ ist dementsprechend ein „Name“, ein sprachlicher Ausdruck für das „Gleichnis der Vollkommenheit“, ebenfalls ein sichtbarer Punkt, der eine anders unbegreifliche Wirklichkeit vor Augen führt:

[...] so haben wir eine Art Vollkommenheit, nach dem menschlichen Begriffe eingerichtet: diese ist nämlich, wenn unsere Sinnen ihre Unvollkommenheit nicht mehr begreifen können, und alsdenn nennen wir diese Gleichniß der Vollkommenheit, mit dem Namen Schönheit. (MENGS 1774:18)

Mengs kreiert mit dem sprachlichen Bild eine Theorie jenseits der begrifflichen Abstraktion. Sie schöpft ihre Präzision und Ausführlichkeit aus der Anschaulichkeit, die sie eben beschreibt. Schönheit nicht als Erinnerung, nicht als Abglanz, sondern als „Gleichniß der Vollkommenheit“ ist eine Figur, die die Anschaulichkeit und Vorstellung zusammenführt, sie ist zugleich eine Form der Beschreibung, die eine sprachliche Bildlichkeit voraussetzt. Darum konnte Helmut Pfotenhauer Mengs’ Gedanken als „die erste moderne deutschsprachige Künstlerschrift“ bezeichnen, und genau das mache „wiederum den literarischen Rang der Gedanken aus.“ (PFOTENHAUER / BERNAUER / MILLER 1995:635) Mengs selbst war sich der Kühnheit und des Provokanten seiner theoretischen Schrift offensichtlich bewusst. Er hielt sich anonym und erwartete, nicht verstanden zu werden, „weil das Schreiben nicht mein Beruf ist, und weil ich mir den Tadel der Schwätzer, welche dieselbe etwa nicht verstehen, ersparen will“ (MENGS 1774:10). Dies war wohl kaum nur ein Zugeständnis der Unsicherheit eines schreibenden Malers. Welch hohen Stellenwert Mengs der stilistischen Wahrheit und sprachlichen Gestaltung von Gedanken zusprach, bezeugt sein ausdrücklicher Wunsch, dass seine Schrift in keine fremde Sprache übersetzt werden möge:

Ich ersuche auch alle Liebhaber dieser Art Schriften, so viel möglich, zu verhüten, dass diese nicht in andre Sprachen übersetzt werde, es sey denn, unter meiner Aufsicht; weil ich gewiß weiß, daß meine darinn angewandten Redensarten 
Gabriela Brudzyńska-Němec

in keiner andern Sprache gebraucht werden können; im Welschen würden sie ganz undeutlich, und im Französischen lächerlich scheinen, und würden den zarten Ohren der gemeinen Schriftsteller, und der Zeitvertreib-Gelehrten ein Greuel werden; denn ich habe geschrieben wie ein Meister mit seinen Schülern redet. (MENGS 1774:11f.)

Ein Verweis auf Rede ist hier mit der Überzeugung verbunden, dass dem sprachlichen Ausdruck selbst, nicht nur dem Gedanken dahinter, eine bedeutungstragende und -gestaltende Kraft zusteht. Wie diese Art über Kunst zu schreiben die Empfindlichkeit des Publikums traf, bezeugt allein die Tatsache, dass von der Erstausgabe der Gedanken über die Schönheit und den Geschmack in der Malerey von Mengs in knapp mehr als einem Jahr 900 Exemplare verkauft wurden, es folgten Neudrucke und Übersetzungen. ${ }^{22}$

\section{„Gehe hin und sieh“}

Winckelmann erkannte in Mengs' beinahe programmatischer begrifflicher Unschärfe jene Leistung des sprachlichen Ausdrucks, die aus der Anschauung der Kunst ihr Wirkungsvermögen schöpfte und in Bezug auf sein Objekt, die bildende Kunst, mehr leistet als die philosophische Ästhetik. Er schrieb an Salomon Geßner am 20. Juni 1761 über Mengs:

[...] der Verfasser hat vermuthlich die Schwierigkeit eines handgreiflichen klaren Begrifs, den niemand hat geben können, eingesehen, und deswegen hat er denselben in ein erhabenes Bild gefasset, welches ich niemals ohne Rührung habe lesen können, und ich preise Gott, der solche Kraft zu denken im Menschen gelegt hat.“ (WINCKELMANN 1778:34)

Das Bildhafte der Sprache, ihre umhüllende Nähe zum Gegenstand, ihre Eigenschaft des doppelten Spiegels oder des leichten Gewands verleiht ihr die Gestalt eines Entwurfs, einer Kontur, die aufzufüllen ist durch die gedankliche Leistung des Lesers, des Betrachters. Sowohl Winckelmann als auch Mengs verstanden ihre Schreibform als eine rednerisch freie, auf die Vervollständigung ausgerichtete Skizzierung, abgeschlossen und doch von kommunikativer Offenheit gekennzeichnet. Mengs resümierte im letzten Teil seiner Gedanken:

Es wäre allein ein Buch von der Bedeutung Raphaels zu schreiben, ich weiß aber das wenige so ich sage, genug ist für die, so selbst nachdenken wollen, für die

22 Vgl. Pfotenhauer / Bernauer / Miller (1995:614): „Für eine Abhandlung zur Kunsttheorie ist ein Verkauf von fast tausend Exemplaren in so kurzer Zeit ein beinahe beispielloser Erfolg.“ 
aber so sich keine Mühe thun viel zu viel schon gesagt ist, und sie, das was ich schreibe nicht begreifen werden [...] (MENGs 1774:100f.)

Und Winckelmann schloss die Abhandlung vor der Fähigkeit der Empfindung des Schönen in der Kunst ab:

Dieses kann zur Absicht dieses Entwurfs, welcher allgemein seyn sollte, hinlänglich geachtet werden. Die höchste Deutlichkeit kann Dingen, die auf der Empfindung bestehen, nicht gegeben werden, und hier läßt sich schriftlich nicht alles lehren [...] gehe hin und sieh [...]. (WINCKELMANN 1968c:233)

\section{Literatur}

Anonym (1763): Mengs, A.R.: Gedanken über die Schönheit und über den Geschmack in der Malerey. Zürich: Heidegger 1762. Rezension. In: Bibliothek der schönen Wissenschaften und der freyen Künste 9:234-257.

ANONYM (1764): Winckelmann, J.J.: Abhandlung von der Fähigkeit der Empfindung des Schönen in der Kunst, und dem Unterrichte in derselben. Dresden: Walther 1763. Rezension. In: Bibliothek der schönen Wissenschaften und der freyen Künste 10,2:251-270.

BÄTSCHMANN, OsKaR (2009): Einführung in die kunstgeschichtliche Hermeneutik. Darmstadt.

Boehm, GotTfried (2005): Das Bild und die hermeneutische Reflexion. In: FigaL, GÜNTER / GANDER, HANs-Helmuth (eds.): „Dimensionen des Hermeneutischen“: Heidegger und Gadamer. Frankfurt (M.), 23-35.

- (2007): Zuwachs an Sein. Hermeneutische Reflexion und bildende Kunst. In: BoenM, GotTFried: Wie Bilder Sinn erzeugen. Die Macht des Zeigens. Berlin, 243-267.

BRUDZYŃSKA-NĚMEC, GABRIELA (2012): Schöpferische Kunstbetrachtung nach Johannes Urzidil. Theoretische Erörterung und literarische Bilder zu „Die Herzogin von Albanera“ und „Der Schauspieler“. In: HöHne, STEFFEN / JoHAnN, KLAus / NĚMEC, MiREK (eds.): Johannes Urzidil. Ein „,hinternationaler“ Schriftsteller zwischen Böhmen und New York. Wien, 243-254.

Goethe, Johann WolfgAng (1998): Ästhetische Schriften 1771-1805. In: Sämtliche Werke, Briefe, Tagebücher und Gespräche. Bd. 18. Hrsg. von Friedmar Apel und Hendrik Birus. Frankfurt (M.).

- (1998a): Winckelmann und sein Jahrhundert. In: Sämtliche Werke, Briefe, Tagebücher und Gespräche. Bd. 19. Hrsg. von Friedmar Apel, Hendrik Birus und Dieter Borchmeyer. Frankfurt (M.), 9-233.

KASE, Oliver (2010): Mit Worten sehen lernen. Bildbeschreibung im 18. Jahrhundert. Petersberg. 
Gabriela Brudzyńska-Němec

Lessing, GotTHOLd EpHRAim (1980): Laokoon oder über die Grenzen der Malerei und Poesie. Stuttgart.

Liessmann, KonRad Paul (2009): Schönheit. Wien.

MENGS, Anton RAPHAEL (1774): Gedanken über die Schönheit und den Geschmack in der Malerey. Zürich.

- (1843): Ueber das „Je ne sais quoi“ in den schönen Künsten. In: SchiLling, GusTAV (ed.): Anton Raphael Mengs' sämmtliche hinterlassene Schriften. Erster Band. Bonn, 251-257.

Moritz, Karl Philipp (1962): Die Signatur des Schönen. In: Schriften zur Ästhetik und Poetik. Hrsg. von Hans Joachim Schrimpf. Tübingen, 93-103.

PANOFSKY, ERWIN (1992): Zum Problem der Beschreibung und Inhaltsdeutung von Werken der bildenden Kunst. In: WeISSTEIN, UlRICH (ed.): Literatur und bildende Kunst. Berlin, 210-220.

Penzel, JoAchim (2007): Der Betrachter ist im Text. Konversation und Lesekultur in deutschen Gemäldegalerien zwischen 1700 und 1914. Berlin.

Pfotenhauer, Helmut (1995): Winckelmann und Heinse: Die Typen der Beschreibungskunst im 18. Jahrhundert oder die Geburt der neueren Kunstgeschichte. In: Boehm, GotTfried / Pfotenhauer, Helmut (eds.): Beschreibungskunst - Kunstbeschreibung: Ekphrasis von der Antike bis zur Gegenwart. München, 313-331.

Pfotenhauer, Helmut / Bernauer, Markus / Miller, Norbert (eds.) (1995): Bibliothek der Kunstliteratur in vier Bänden - Band 2: Frühklassizismus. Position und Opposition: Winckelmann, Mengs, Heinse. Frankfurt (M.).

RÖTtGen, STEFFI (1986): Winckelmann, Mengs und die deutsche Kunst. In: GaEHTGENS, THOMAs W. (ed.): Johann Joachim Winckelmann 1717-1768. Hamburg, 161-178.

SCHRÖTER, ELISABETH (1986): Winckelmanns Projekt einer Beschreibung der Altertümer in den Villen und Palästen Roms. In: GaEhtgens, Thomas W. (ed.): Johann Joachim Winckelmann 1717-1768. Hamburg, 55-119.

SutTer, MONIKA (1968): Die kunsttheoretischen Begriffe des Malerphilosophen Anton Raphael Mengs. München [Diss.].

TATARKIEWICZ, WŁadYSŁAW (2003): Geschichte der sechs Begriffe: Kunst · Schönheit $\cdot$ Form $\cdot$ Kreativität $\cdot$ Mimesis $\cdot$ Ästhetisches Erlebnis. Aus dem Polnischen von Friedrich Griese. Frankfurt (M.) [Originalausgabe Warszawa 1976].

Trautwein, RoBert (1997): Geschichte der Kunstbetrachtung: von der Norm zur Freiheit des Blicks. Köln.

UEDING, GERT (1995): Von der Rhetorik zur Ästhetik. Winckelmanns Begriff des Schönen. In: RAULET, GÉRARD (ed.): Von der Rhetorik zur Ästhetik. Studien zur Entstehung der modernen Ästhetik im 18. Jahrhundert. Rennes, 41-66.

WALZEL, OsKaR (1917): Wechselseitige Erhellung der Künste. Ein Beitrag zur Würdigung kunstgeschichtlicher Begriffe. Berlin. 
Überlegungen nach Anton Raphael Mengs und Johann Joachim Winckelmann

WincKelmann, Johann JoAchim (1764): Geschichte der Kunst des Alterthums. Erster Theil. Dresden.

- (1778): Winckelmanns Briefe an seine Freunde in der Schweiz. Zürich.

- (1968): Gedancken über die Nachahmung der Griechischen Wercke in der Mahlerey und Bildhauer-Kunst. In: Kleine Schriften, Vorreden, Entwürfe. Hrsg. von Walther Rehm. Berlin, 27-59.

- (1968a): Erinnerung über die Betrachtung der Werke der Kunst. In: Kleine Schriften, Vorreden, Entwürfe. Hrsg. von Walther Rehm. Berlin, 149-156.

- (1968b): Beschreibung des Torso im Belvedere zu Rom. In: Kleine Schriften, Vorreden, Entwürfe. Hrsg. von Walther Rehm. Berlin, 169-173.

- (1968c): Abhandlung von der Fähigkeit der Empfindung des Schönen in der Kunst, und dem Unterrichte in derselben. In: Kleine Schriften, Vorreden, Entwürfe. Hrsg. von Walther Rehm. Berlin, 211-233.

- (1968d): Vorrede zu der Geschichte der Kunst des Alterthums. In: Kleine Schriften, Vorreden, Entwürfe. Hrsg. von Walther Rehm. Berlin, 235-246.

ZEDLER, JOHANN HEINRICH (ed.) (1732-1754): Großes vollständiges Universallexikon aller Wissenschaften und Künste. Leipzig/Halle. 